\title{
Kinematics of Solar Chromospheric Surges of AR 10930
}

\author{
Su-Chan Bong ${ }^{1,2}$, Kyung-Suk Cho ${ }^{1,2}$, And VAsyl Yurchyshyn ${ }^{3,1}$ \\ ${ }^{1}$ Korea Astronomy and Space Science Institute, 776 Daedeokdae-ro, Yuseong-gu, Daejeon 305-348, Korea \\ scbong@kasi.re.kr, kscho@kasi.re.kr \\ ${ }^{2}$ University of Science and Technology, 217 Gajeong-ro, Yuseong-gu, Daejeon 305-333, Korea \\ ${ }^{3}$ Big Bear Solar Observatory, New Jersey Institute of Technology, 40386, North Shore Lane, Big Bear City, CA 92314-9672, \\ USA; vayur@bbso.njit.edu
}

Received November 11, 2014; accepted December 6, 2014

\begin{abstract}
Solar chromospheric surges are often reported to contain rotational motion. However, the details of the motion and driving mechanism of the surges are not yet fully understood. Recurrent surges with rotational motion at AR 10930 on the west limb are observed by Hinode Solar Optical Telescope (SOT) continuously from 11:21 UT on December 18 to 09:58 UT on December 19, 2006, using the Ca II H broadband filter. We analyze details of the motion including number of turns from the rise of the surge to the fall, axial speed and acceleration. During the observation, rise and fall motion accompanying rotation appears recurrently. There occur a total of 14 surges at AR 10930 over 17 hours. The average duration is 45 minutes, and the average width, and length are $8 \mathrm{Mm}$, and $39 \mathrm{Mm}$, respectively. We speculate that the surges occurred by recurrent reconnections between the twisted prominence and large untwisted flux tube.
\end{abstract}

Key words: Sun: chromosphere — Sun: filaments, prominences — Sun: magnetic topology

\section{INTRODUCTION}

Surges are small scale eruptions of cool material in the solar atmosphere, which are usually observed in chromospheric lines such as $\mathrm{H} \alpha$. Since their dynamics represents the motion of plasma along magnetic field lines, surges are useful to study the magnetic field structure and its change.

It has been reported that surges rise upwards then diffuse, fade out, and often fall back down. They also often exhibit helical motion around their axis of ejection (Bruzek 1974). Helicity pumping, convective vortex, and reconnection have been proposed as driving forces of the motion. Parker (1974) suggested that when part of a twisted flux tube expands, a torque is produced that untwists the unexpanded part. By inspecting photospheric strong shear flows and Yohkoh soft X-ray images, Chae et al. (2003) showed that an $\mathrm{X}$-ray loop in the corona actually expanded while magnetic helicity was pumped in by the photospheric transient shear flows. Meanwhile, Velli \& Liewer (1999) proposed convectively driven vortex flows in the photosphere as a source of the helical motion. More recently, Wedemeyer-Böhm et al. (2012) discovered small scale swirling motions $\left(<10^{\prime \prime}\right)$ in the chromosphere, which are located above magnetic flux concentrations formed by converging and sinking plasma in the photosphere. They noted that the cross section of the swirl increased with height. Using 2.5-dimensional MHD simulations, Shibata \& Uchida (1986) proposed that an eruption of an untwisting magnetic jet (relaxation process) can be produced by reconnection between a twisted loop and an open flux tube. Unwinding of helical structures were

Corresponding Author: S.-C. Bong reported in case of UV jets (Liu et al. 2011), chromospheric spicules (Sterling et al. 2010), and $\mathrm{H} \alpha$ surges (Canfield et al. 1996). The interpretation is that the twist of an emerging flux is transferred via the interchange reconnection. However, details of the motion and the driving mechanism are not fully understood. We revisit this issue utilizing a high resolution data set obtained by Hinode Solar Optical Telescope (SOT) instrument (Kosugi et al. 2007; Tsuneta et al. 2008).

Hinode SOT observed the west limb of the Sun with high spatial and temporal resolution for about 23 hours from 11:21 UT on December 18 to 09:58 UT on December 19, 2006. During that time, 14 recurrent surges were identified, which makes it a unique data set for studying the dynamics of surges. These showed upward and downward motions as well as accompanying rotations. In this study, we analyze their dynamic properties including number of turns, the axial speed, and the acceleration.

\section{DATA AND REDUCtion}

Figure 1 shows evolution of NOAA AR 10930 as recorded by TRACE satellite at the $195 \AA$ line. According to Park et al. (2010), this active region produced several flares with the injection of a large amount of helicity. However neither strong flares nor large scale eruptions were reported after December 18.

These surges were well observed by SOT, onboard Hinode satellite. Usually chromospheric surges are observed in the $\mathrm{H} \alpha$ line on the ground. The data obtained from ground-based instruments are impaired by atmospheric seeing distortion. The SOT is the largest and the most advanced space borne solar telescope, virtu- 


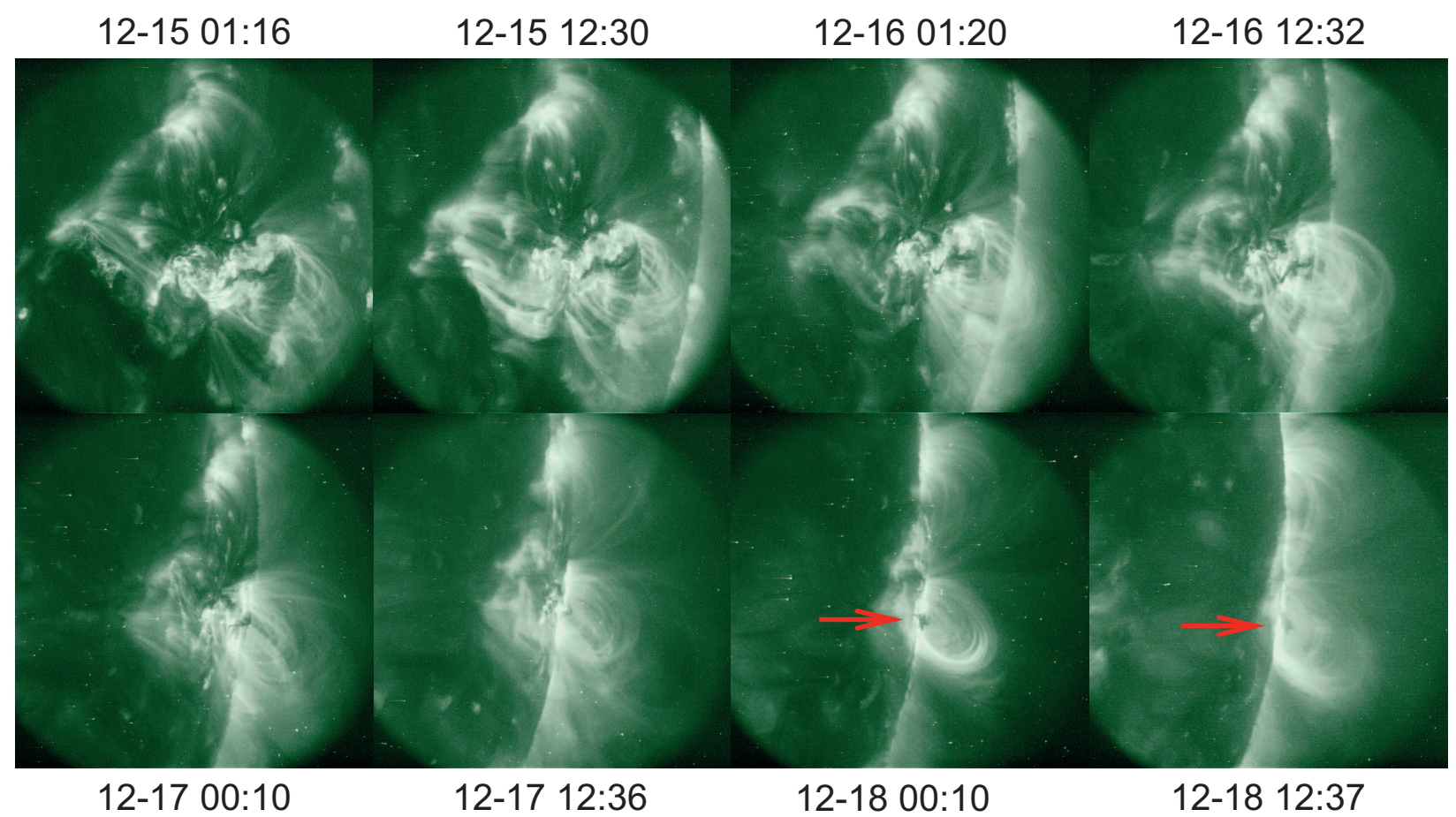

Figure 1. Evolution of the Active Region 10930 observed by TRACE satellite at the $195 \AA$ line from December 15 to December 18, 2006. A prominence appeared on December 18 as denoted by a red arrow.

ally free of distortion. It consists of a $0.5 \mathrm{~m}$ diameter Gregorian telescope and a focal plane package (Suematsu et al. 2008; Tsuneta et al. 2008). Therefore, the SOT offers excellent opportunity to study the dynamics of chromospheric surges. Data from SOT were obtained with a temporal cadence of $8 \mathrm{sec}$ and high spatial resolution of $0.2^{\prime \prime}$ using the Ca II H filter. We used 10029 images with a field of view of $110^{\prime \prime}$ by $110^{\prime \prime}$ centered on the west limb near the equator $\left(1000^{\prime \prime},-100^{\prime \prime}\right)$.

Standard SSW procedure (FG_PREP.PRO) was applied to correct camera readout defects and to remove radiation-belt/cosmic ray spikes. We then aligned all the images by identifying limb features adopting the theoretical limb window, and applying the correlation tracking technique to the limb feature. This resulted in a 23 hour long movie, and we identified the following mass motions: (1) spicules and filaments, (2) downflows from the corona, (3) macro-spicules, (4) horizontal motions in filaments, and (5) recurrent surges.

\section{AnAlysis AND Results}

\subsection{Typical Motion of Recurrent Surges}

Figure 2 shows a typical example of a recurrent surge, which could be identified 14 times during 23 consecutive hours. They first move upward at an angle and then fall back. Detailed inspection of the motion of the surges from the movie reveals that they rotate rapidly as they rise, while they only show weakly rotating and straight motions when they fall. In the figure, the upper panels show a surge during the rise and the lower panels during the fall. The white arrow indicates the rotating tip of the surge as it rises. The green and yellow arrows mark dense falling masses. Near the foot of the surge, there is a filament containing horizontal flows. It appears likely that some of the filament material moves together with the surge. In the lower panels, we could identify a void, which is absent in upper panel under the filament $\left(978^{\prime \prime}\right.$, $\left.-130^{\prime \prime}\right)$. This implies that the filament might provide material to the surge, however the connection between the surge and filament is not clear.

\subsection{Surge Dynamics}

To quantitatively analyze plasma flows, we construct a time-slice plot. We manually determined the axis of the surge and the left and right boundaries as marked with plus symbols shown in Figure 3. The width of the surge in the figure is about $6 \mathrm{Mm}$. The projected axis was slanted from the vertical by 60 degree. In each frame, we averaged the intensity within the boundary in the direction perpendicular to the axis. The upper panel of Figure 4 shows the time-slice plot made by stacking the averaged intensity from 16:37 UT to 17:22 UT on December 18. In this figure we can see the change of the axial distance of the surge with many rising and falling features. We focus on the feature which reached the highest position. It began to rise rapidly after 16:40 UT, reached the highest position of $39 \mathrm{Mm}$ at 16:54 UT and fell down as outlined by bright features. The thick solid line is a smooth curve representing the top boundary of the surge. It was determined manually by selecting several points and using spline interpolation. To inspect the rotational motion of the top boundary in detail, in the lower panel of Figure 4 we plot the intensity in the direction perpendicular to the surge axis 


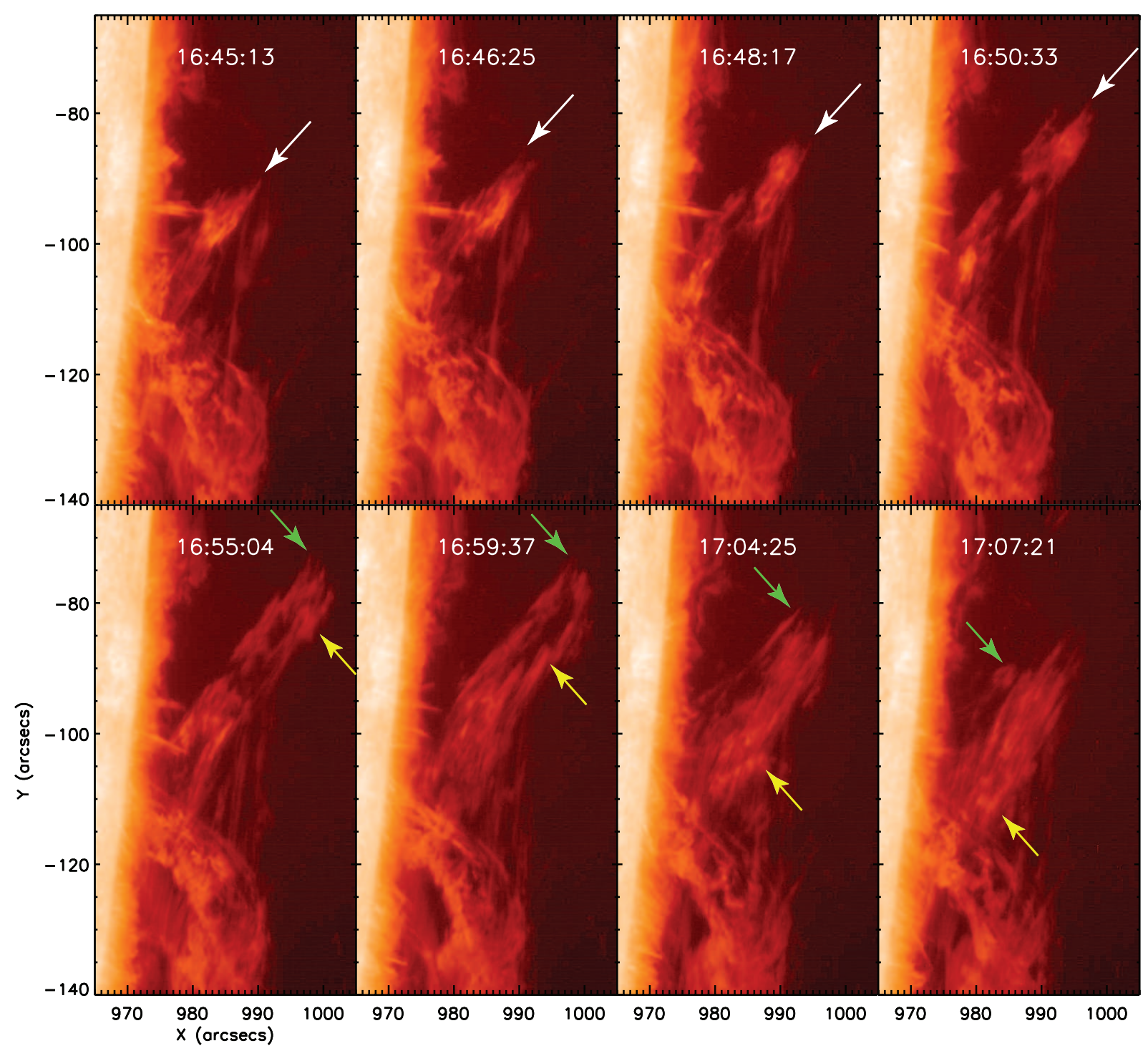

Figure 2. A typical example of the 14 surges and prominence observed in Ca II H line by Hinode SOT on December $18,2006$. The surge showed rapid rotating motion (white arrow) when rising and then plasma was falling back along a straight line (green and yellow arrows).

at the axial distance changing with time following the black line in the upper panel.

In the beginning, the surge shows rapid rotational motions from right (positive value) to left (negative value) direction overlapping with those in opposite direction during 16:37 UT - 16:44 UT. The estimated maximum cross axial speed is approximately $20 \mathrm{~km} \mathrm{~s}^{-1}$. Similar but more discernible and decelerating motion can be seen from 16:45 UT to 16:54 UT, which changes to spreading rather than rotational flows after 16:54 UT, suggesting that the structure rotated rapidly at the beginning, then slowed down during the rise phase, and spread out as it fell. The number of the rotations from the start to the end seems to be at least three.

Figure 5 shows the axial velocity and acceleration of the top of the surge. The axial mean speed during the rising phase is about $30 \mathrm{~km}^{-1}$, and the speed during the falling phase is roughly $20 \mathrm{~km}^{-1}$. We found that the velocity reaches up to $\sim 40 \mathrm{~km} \mathrm{~s}^{-1}$ within the first 10 minutes after the onset of the surge. The estimated acceleration exceeds $100 \mathrm{~m} \mathrm{~s}^{-2}$ during the period. By assuming typical values for the density $\left(5 \times 10^{-11} \mathrm{~kg} \mathrm{~m}^{-3}\right)$ and magnetic field strength $(5 \mathrm{G})$ of quiescent prominences (Soler et al. 2010), the estimated Alfvén speed is $63 \mathrm{~km} \mathrm{~s}^{-1}$, which is of the same order but larger than the maximum ascending speed. This is understandable if the twist propagates upward in the form of a torsional Alfvén wave while the material is retarded through the rotational motion. After the speed peaked, it began to decrease steadily at a constant deceleration rate of $70 \mathrm{~m} \mathrm{~s}^{-2}$, which is smaller than the gravitational acceleration of the Sun along the slanted axis (60 degree from the normal). This slow fall may be due to support by some force or just a projection effect. If the latter, the actual angle of the axis should be 75 degree from the normal. 


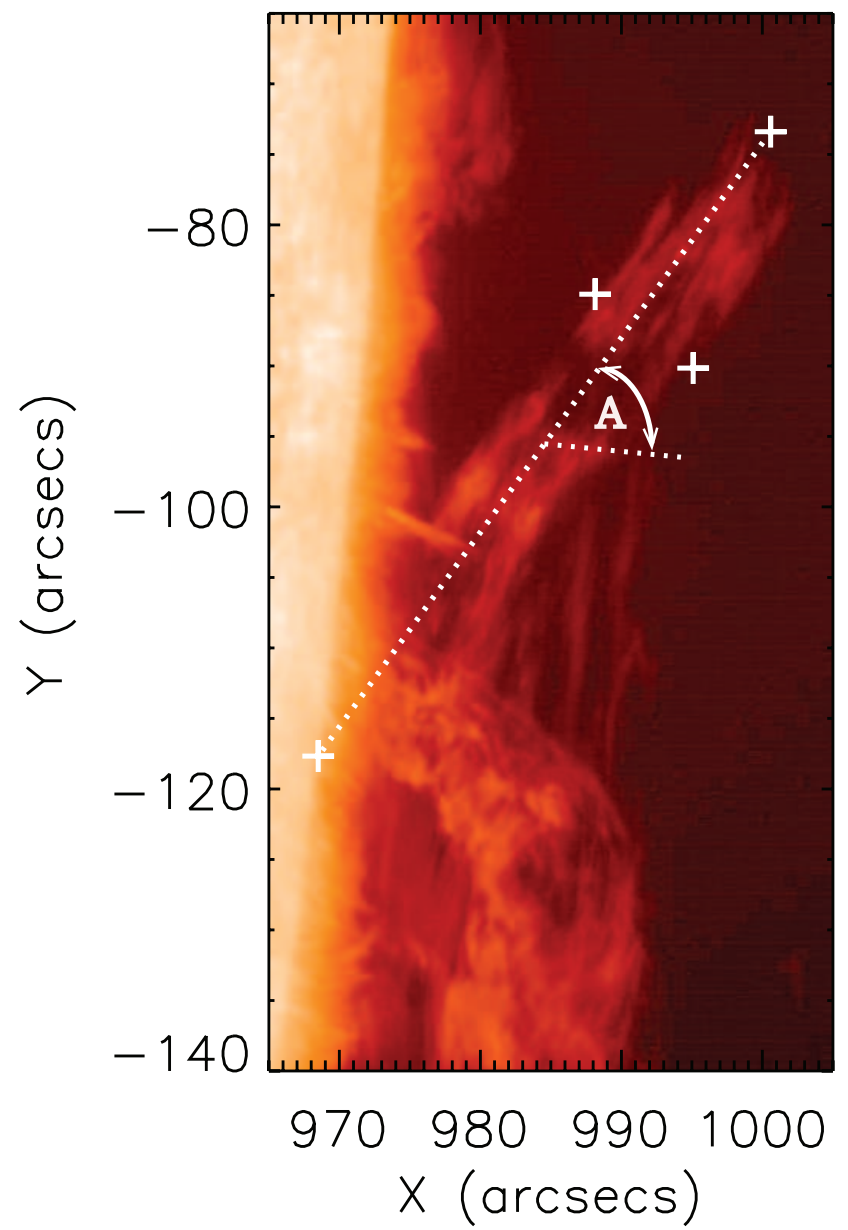

Figure 3. A measurement of properties of a surge. Width and length of the surge are measured manually as illustrated by plus symbols. 'A' denote the zenith angle of the axis.

Specific kinetic energy $\left(v^{2} / 2\right)$ deduced from the rotational motion (cross axial velocity) at initial phase is about $2 \times 10^{8} \mathrm{~J} \mathrm{~kg}^{-1}$, and that from the axial motion after the onset is about $8 \times 10^{8} \mathrm{~J} \mathrm{~kg}^{-1}$. These values are significantly less than the maximum specific gravitational potential energy increase $\left(3 \times 10^{9} \mathrm{~J} \mathrm{~kg}^{-1}\right)$ at the maximum height. This means that the energy for the surge motion was somewhat continuously supplied rather than impulsively, more energy being added during the rising phase.

\subsection{Physical Properties}

We analyzed 14 surges that recurrently rise and fall with similar untwisting motion. The surge footpoint gradually moves southward, while the southern filament dismantled as shown in Figure 6. The solid lines represent the axis orientation and length, while colors represent peak times of the surges. The duration and morphology of the surges are summarized in Table 1. Each event is defined from the start of the rising motion to the end of the falling motion until it is indistinguishable. We determine the peak time when the surge reaches the highest point. The length, width, and axis
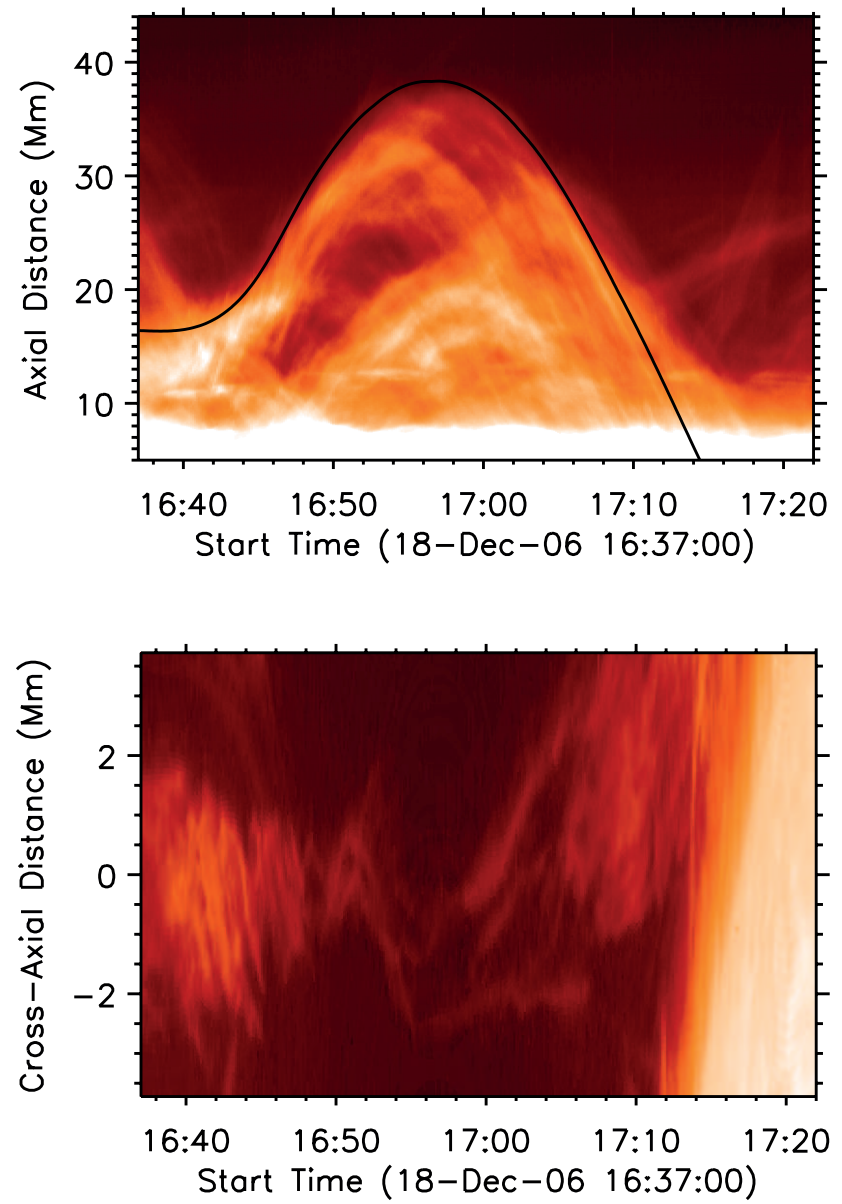

Figure 4. Motion of a surge along the axis (upper panel) and across the axis top (bottom panel). To produce the upper space time plot we averaged intensity over the width for 6 $\mathrm{Mm}$. The height of the surge is indicated by a black line. Lower panel shows intensity change along a cross-axial cut made at the top position of the surge.

angle are measured at the peak time as shown in Figure 3. The length is measured from the peak point to the apparent disk limb along the axis (dotted line). Width is the cross axial distance, and the zenith angle is the measured angle between the axis and the radial direction from the solar center. As follows from Figure 6 and Table 1, the surges show a broad range of sizes and durations, while they have similar axis inclination angle. Their estimated average duration, width, and length are 45 minutes, $8 \mathrm{Mm}$, and $39 \mathrm{Mm}$, respectively. We show a scatter plot of the width and length in upper panel of Figure 7. It shows that the length is roughly proportional to the width but with large scatter. The ascending and descending times are correlated with the length as shown in the middle and lower panels, which means that on the average, rising speeds are similar to each other and the same applies to the falling speeds. It looks likely that the speed of ascent is faster than while descending. 
Table 1

Duration and morphology of the surges

\begin{tabular}{ccccccccc}
\hline Number & $\begin{array}{c}\text { Date } \\
(\mathrm{UT})\end{array}$ & $\begin{array}{c}\text { Start } \\
(\mathrm{UT})\end{array}$ & $\begin{array}{c}\text { Peak } \\
(\mathrm{UT})\end{array}$ & $\begin{array}{c}\text { End } \\
(\mathrm{UT})\end{array}$ & $\begin{array}{c}\text { Duration } \\
(\text { minute })\end{array}$ & $\begin{array}{c}\text { Width } \\
(\mathrm{Mm})\end{array}$ & $\begin{array}{c}\text { Length } \\
(\mathrm{Mm})\end{array}$ & $\begin{array}{c}\text { Angle } \\
\text { (degree) }\end{array}$ \\
\hline 1 & $2006-12-18$ & $12: 31$ & $12: 40$ & $12: 59$ & 28 & 5 & 24 & 44 \\
2 & $2006-12-18$ & $14: 03$ & $14: 17$ & $14: 24$ & 21 & 2 & 17 & 40 \\
3 & $2006-12-18$ & $14: 28$ & $15: 19$ & $15: 59$ & 91 & 12 & 54 & 54 \\
4 & $2006-12-18$ & $16: 08$ & $16: 30$ & $16: 47$ & 39 & 6 & 47 & 67 \\
5 & $2006-12-18$ & $16: 37$ & $16: 54$ & $17: 22$ & 45 & 6 & 39 & 60 \\
6 & $2006-12-18$ & $17: 05$ & $17: 24$ & $17: 47$ & 42 & 13 & 53 & 65 \\
7 & $2006-12-18$ & $17: 41$ & $18: 08$ & $18: 43$ & 62 & 7 & 54 & 66 \\
8 & $2006-12-18$ & $18: 28$ & $18: 42$ & $18: 58$ & 30 & 7 & 35 & 58 \\
9 & $2006-12-18$ & $19: 31$ & $19: 46$ & $20: 10$ & 39 & 7 & 41 & 58 \\
10 & $2006-12-18$ & $21: 09$ & $21: 26$ & $21: 52$ & 43 & 6 & 25 & 73 \\
11 & $2006-12-19$ & $00: 15$ & $00: 31$ & $00: 53$ & 38 & 6 & 33 & 53 \\
12 & $2006-12-19$ & $01: 19$ & $01: 49$ & $02: 17$ & 58 & 9 & 43 & 73 \\
13 & $2006-12-19$ & $02: 55$ & $03: 17$ & $03: 55$ & 60 & 13 & 47 & 59 \\
14 & $2006-12-19$ & $04: 38$ & $04: 54$ & $05: 10$ & 32 & 10 & 32 & 57 \\
\hline Average & & & & & 45 & 8 & 39 & 59 \\
\hline
\end{tabular}

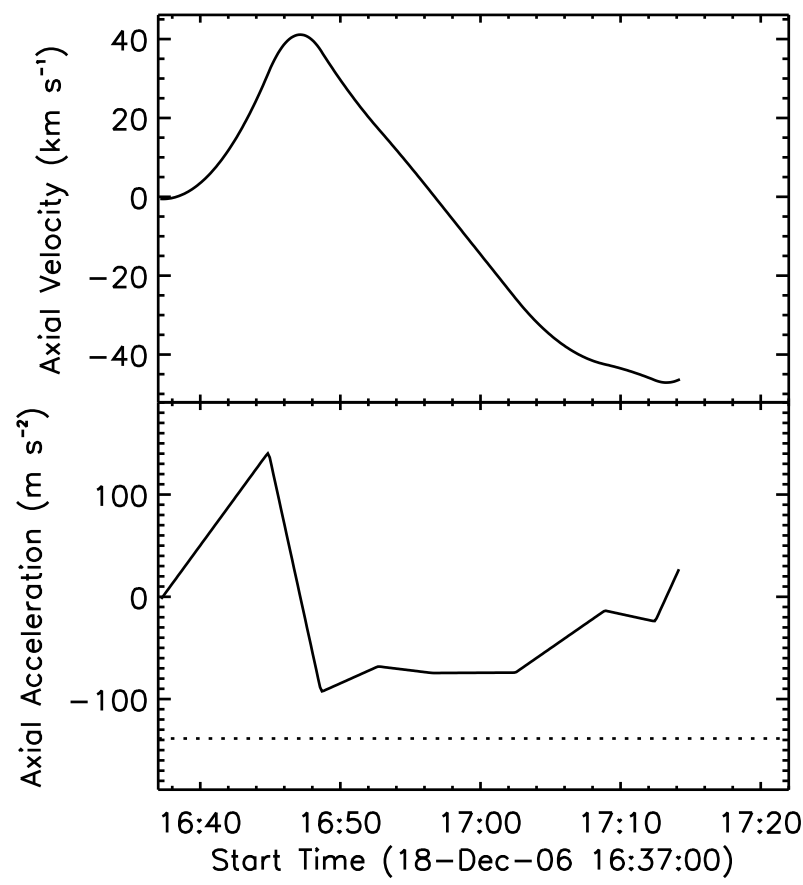

Figure 5. Time profiles of the projected axial velocity and acceleration. Dotted line is the gravitational acceleration of the Sun.

\section{Discussion ANd Conclusion}

We analyzed chromospheric recurrent surges observed by Hinode SOT with the Ca II H filter. We could identify 14 surges over a 17 hour interval. They all show fast rotational motion at the beginning, which slowed as the surges rose, and then fell with much slower or no rotation. Their width varied from 2 to $13 \mathrm{Mm}$, lengths from 17 to $54 \mathrm{Mm}$, and life time from 21 to 91 minutes, with average values of $8 \mathrm{Mm}, 39 \mathrm{Mm}$, and 45 minutes respectively. Their projected axes on the plane of sky

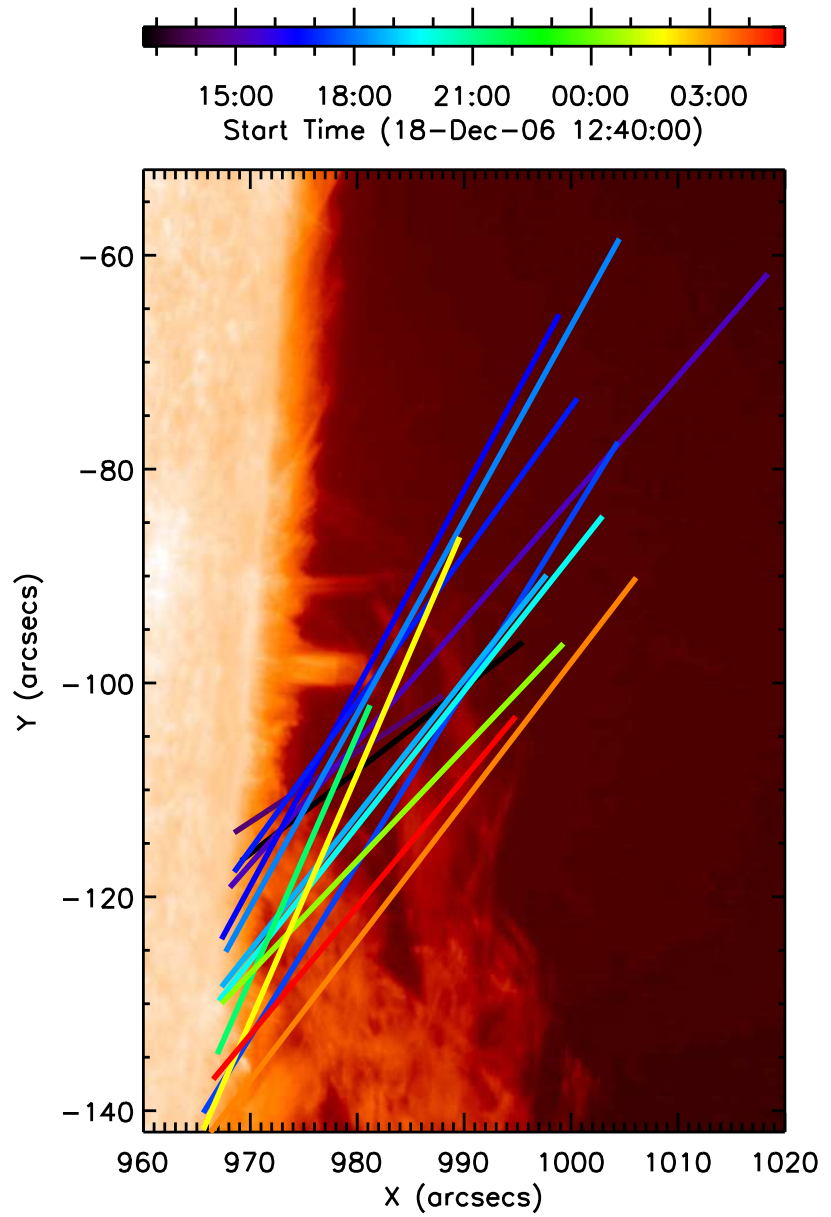

Figure 6. Length and position of axes of 14 surges. Color change represents time. Directions of the surge were similar in all cases, but their lengths are different.

were similar to one another within 33 degrees, although the origin of each trajectory was slightly shifting southward while the nearby filament was disappearing. We 

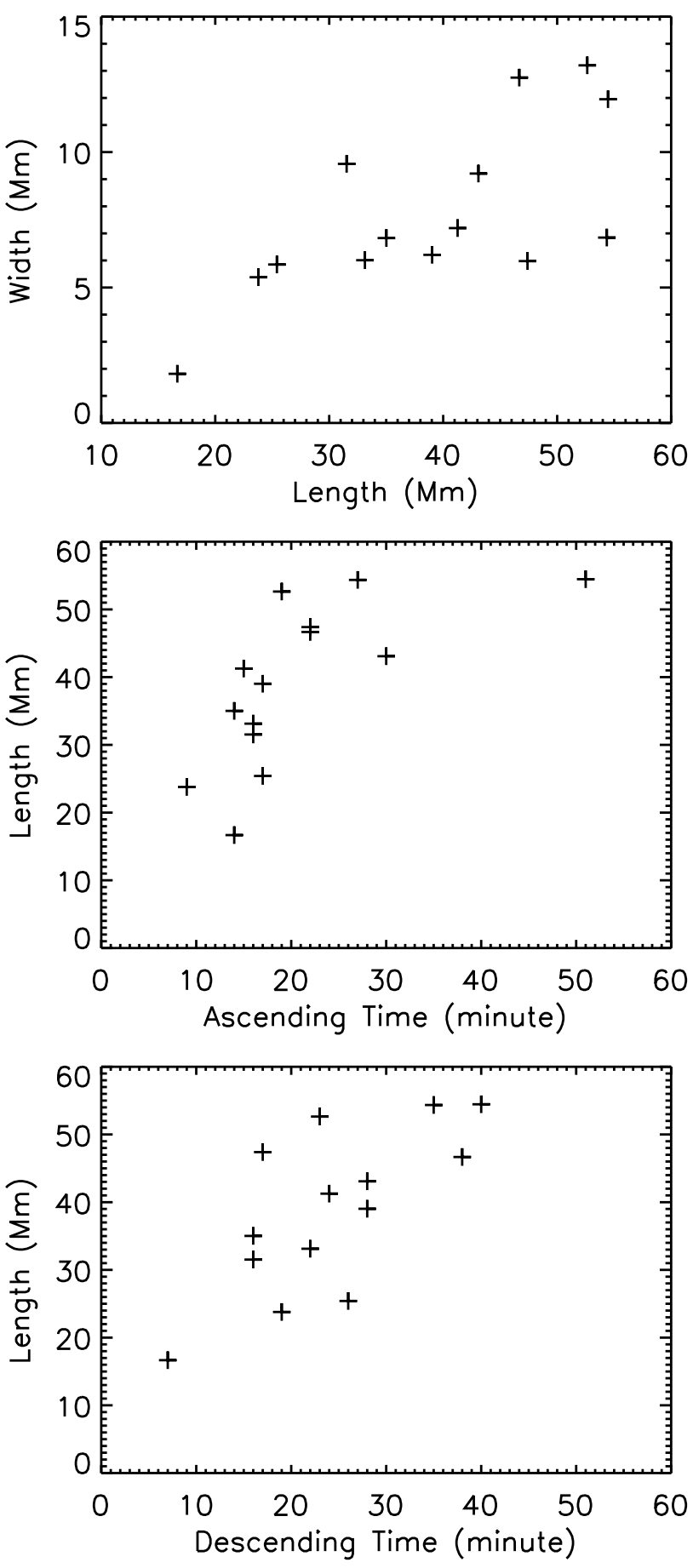

Figure 7. Correlations of length with width (upper panel), ascending time with length (middle panel), and descending time with length (lower panel).

also found that the kinetic energy of the initial rotational motion is significantly less than the maximum gravitational potential energy, which implies that the energy input was rather gradual than impulsive.

We speculate on three possible mechanisms for triggering the rotational motion. The first is the 'helicity pumping' proposed by Parker (1974) and observationally supported by Chae et al. (2003). In this case, the helicity within a twisted flux tube is moved from the unexpanded segment to the expanded segment, causing twist-loosening motion in the unexpanded segment. As the chromospheric surges in this study show twistloosening motions, there should be significant expansion in the other part of the flux tube in the high corona. However, we could not find any expansion or significant change of coronal loops from TRACE observation during the event period.

The second possibility is the 'convective vortex' mechanism suggested by Velli \& Liewer (1999). According to them, a rotation could be generated by convectively driven photospheric vortices. The 'convective vortex' would have an observational feature of a rotational motion in the photosphere. The scale of the rotational motion is about $8 \mathrm{Mm}$ in this study, which is about 10 times the granule size. We can hardly expect a rotational motion in the photosphere with an $8 \mathrm{Mm}$ diameter within 20 minutes, which implies a rotational speed of $20 \mathrm{~km} \mathrm{~s}^{-1}$. Even if the observed rotational motion originates from a small scale motion in the photosphere, it should expand rapidly with height. However, we could not see any structures expanding from the footpoints of the surges upward, and it looks likely that the falling material was spreading out rather than converging.

The last mechanism is the 'reconnecting untwisting magnetic jet' proposed by Shibata \& Uchida (1986) in their 2.5-dimensional MHD simulation. In this case, the footpoints of the magnetic field lines are assumed to be fixed on the photosphere. Reconnection of a large and weakly twisted flux with a small and strongly twisted (emerging) flux or a filament can trigger the transfer of helicity from the twisted to the untwisted system. The observational properties such as a large scale rotational motion without expansion are well explained by their scenario, however we could not find any evidence of a reconnection supporting this scenario. Further detailed investigation is required to explain the large number (more than three) of the turns of surges even at the relatively low height. The number of turns in a stable flux tube is generally expected less than 1.75 under coronal conditions (Kumar et al. 2012). Therefore some stabilizing mechanism should have suppressed the kink instability of the small and strongly twisted flux tube until the reconnection or flux emergence occurs.

\section{ACKNOWLEDGMents}

This work was supported by the Management of the Space Weather Prediction Center of KASI and the KASI basic research funds. Hinode is a Japanese mission developed and launched by ISAS/JAXA, with NAOJ as domestic partner and NASA and STFC (UK) as international partners. It is operated by these agencies in co-operation with ESA and NSC (Norway).

\section{REFERENCES}

Bruzek, A. 1974, in IAU Symp. 57, Coronal Disturbances, ed. G. A. Newkirk (Boston: Reidel), 323 
Canfield, R. C., Reardon, K. P., Leka, K. D., Shibata, K., Yokoyama, T., \& Shimojo, M. 1996, H alpha Surges and X-Ray Jets in AR 7260, ApJ, 464, 1016

Chae, J., Moon, Y.-J., Rust, D. M., Wang, H., \& Goode, P. R. 2003, Magnetic Helicity Pumping by Twisted Flux Tube Expansion, JKAS, 36, 33

Kosugi, T., Matsuzaki, K., Sakao, T., et al. 2007, The Hinode (Solar-B) Mission: An Overview, Sol. Phys., 243, 3

Kumar, P., Cho, K.-S., Bong, S.-C., Park, S.-H., \& Kim, Y. H. 2012, Initiation of Coronal Mass Ejection and Associated Flare Caused by Helical Kink Instability Observed by SDO/AIA, ApJ, 746, 67

Liu, C., Deng, N., Liu, R., Ugarte-Urra, I., Wang, S., \& Wang, H. 2011, A Standard-to-Blowout Jet, ApJ, 735, L18

Park, S.-H., Chae, J., Jing, J., Tan, C., \& Wang, H. 2010, Time Evolution of Coronal Magnetic Helicity in the Flaring Active Region NOAA 10930, ApJ, 720, 1102

Parker, E. N. 1974, The Dynamical Properties of Twisted Ropes of Magnetic Field and the Vigor of New Active Regions on the Sun, ApJ, 191, 245

Shibata, K., \& Uchida, Y. 1986, Sweeping-Magnetic-Twist
Mechanism for the Acceleration of Jets in the Solar Atmosphere, Sol. Phys., 103, 299

Soler, R., Arregui, I., Oliver, R., \& Ballester, J. L. 2010, Seismology of Standing Kink Oscillations of Solar Prominence Fine Structures, ApJ, 722, 1778

Sterling, A. C., Harra, L. K., \& Moore, R. L. 2010, Fibrillar Chromospheric Spicule-Like Counterparts to an ExtremeUltraviolet and Soft X-Ray Blowout Coronal Jet, ApJ, 772,1644

Suematsu, Y., Tsuneta, S., Ichimoto, K., et al. 2008, The Solar Optical Telescope of Solar-B (Hinode): The Optical Telescope Assembly, Sol. Phys., 249, 197

Tsuneta, S., Ichimoto, K., Katsukawa, Y., et al. 2008, The Solar Optical Telescope for the Hinode Mission: An Overview, Sol. Phys., 249, 167

Velli, M., \& Liewer, P. 1999, Alfvén Wave Generation in Photospheric Vortex Filaments, Macrospicules, and "Solar Tornadoes", Space Sci. Rev., 87, 339

Wedemeyer-Böhm, S., Scullion, E., Steiner, O., van der Voort, L. R., de La Cruz Rodriguez, J., Fedun, V., \& Erdélyi, R. 2012, Magnetic Tornadoes as Energy Channels into the Solar Corona, Nature, 486, 505 